\title{
Clinical performance of non-precious metal double crowns with friction pins in severely reduced dentitions
}

\author{
Sebastian Hinz ${ }^{1} \cdot$ Ramona Schweyen $^{1}$ (i) $\cdot$ Jeremias Hey ${ }^{1} \cdot$ Juergen Setz $^{1} \cdot$ Christin Arnold $^{1}$
}

Received: 18 February 2019 / Accepted: 24 January 2020 / Published online: 1 February 2020

(C) The Author(s) 2020

\begin{abstract}
Objectives Several in vitro studies have investigated the retention of double crowns with friction pins (DCP); however, clinical data on their long-term success have not been reported. We sought to evaluate the 5-year survival rate of DCPs in patients with severely reduced dentition (SRD) and not severely reduced dentition (NSRD).

Materials and methods A total of 158 patients were treated with 182 dentures on 520 abutment teeth between 2006 and 2016. The SRD group included 144 dentures that had been inserted on 314 abutment teeth. We evaluated the influence of age, sex, jaw, number, tooth vitality, and abutment teeth localization (according to Steffel's classification) on the 60-month survival rates of dentures and abutment teeth using the Kaplan-Meier estimator, logrank test, and Cox regression.

Results The cumulative 60-month survival rate was $84.3 \%$ (CI 77.1-91.5\%) for all dentures; however, the survival rate in the SRD group $(80.3 \%$; CI $71.5-89.1 \%)$ was significantly lower than in the NSRD group $(100 \% ; p=0.04)$. Dentures classified in Steffel's class A had the lowest survival rate (51.5\%; CI 30.9-72.1\%). Number, location, and vitality of the abutment teeth had a significant impact on survival rate.

Conclusions DCP dentures showed comparable clinical long-term success to double crown systems that have been previously reported in the literature. The number, localization, and vitality of abutment teeth had the greatest influence on the survival rates of denture and abutment teeth.

Clinical relevance DCP dentures have an acceptable 5-year survival rate. Clinical treatment planning must take into account key factors associated with the prognosis of the abutment teeth.
\end{abstract}

Keywords Partial denture $\cdot$ Double crowns $\cdot$ Friction pins $\cdot$ Survival rate $\cdot$ Severely reduced dentition

\section{Introduction}

Double crowns are characterized as a universal attachment that combines various functions, such as support, retention, non-tilt, and shear interlock. As rigid attachments, they transmit all loads (axial, horizontal, and torque) directly to the abutment teeth [1-3]. Different types of double crowns are used for the retention of partial dentures in different countries [1, 4-9]. In addition, cases with significantly reduced residual dentitions and an unfavorable distribution of supporting teeth

Ramona Schweyen

ramona.schweyen@medizin.uni-halle.de

1 Department of Prosthetic Dentistry, University School of Dental Medicine, Martin-Luther-University Halle-Wittenberg, Magdeburger Str. 16, 06112 Halle, Germany have shown high survival rates using double crown systems, suggesting that prosthetic rehabilitation with double crowns can be used in patients with an unfavorable distribution of remaining teeth $[10,11]$.

Severely reduced dentition (SRD) is characterized by less than four teeth in one jaw. Dentures can be dislocated due to chewing forces, which are affected by the number and localization of the abutment teeth: as the number of abutment teeth declines, denture support also declines [12]. This support and the resulting kinetics can influence the stability of the denture position and patients' satisfaction. The long-term behavior of different double crown systems has been evaluated in several clinical studies [13]. Denture retention to the attachment is achieved by static and dynamic friction, which is dependent on double crown type. Sufficient friction is essential for effective denture functioning and patient satisfaction. The most common types of double crowns are telescopic crowns, which provide parallel sidewalls with a convergence very close to $0^{\circ}$ 
and conical crowns with tapered sidewalls. In telescopic crowns, retention is achieved by the large contact surface between the primary and secondary crowns. In conical crowns, retention is achieved by a cone or press fit, depending on the material parameters, cone angle, and joining force [1].

In vitro studies have shown a loss of retention in telescopic and conical crowns after simulated artificial aging [1, 14-16]. Often, the only clinical treatment option in such cases is the fabrication of a new prosthesis. In cases of crowns with additional friction pins, retention is achieved via additional retention elements consisting of prefabricated precision components that can be embedded elastically or locked between the primary and secondary crowns [1] (Fig. 1). This system allows for the individual adjustment of retention forces.

The primary crown is often fabricated with a $2^{\circ}$ groove angle. Primary and secondary crowns provide a tension-free fit. Using a spark erosion process, an insertion groove $\left(0^{\circ}\right)$ is placed in one approximal surface of the primary crown. The corresponding friction pin $(\varnothing=0.7-0.9 \mathrm{~mm})$ produces static friction and is fixed within the secondary crown by laser welding [17-21]. Double crowns with additional friction pins include the secondary crown as part of the denture framework, and both parts are often manufactured as a single cobaltchrome-molybdenum piece, which is biocompatible and hypoallergenic [17-21].

To the best of our knowledge, no studies have evaluated the long-term clinical behavior of double crowns with friction pins (DCP). The aim of the present study was to evaluate the survival time of dentures fixed with DCP and abutment teeth in cases of SRD.

\section{Materials and methods}

\section{Patients}

Patients who received DCP dentures from January 1, 2006 to January 31, 2016 at the Department for Prosthodontics at the Martin-Luther-University Halle-Wittenberg were included

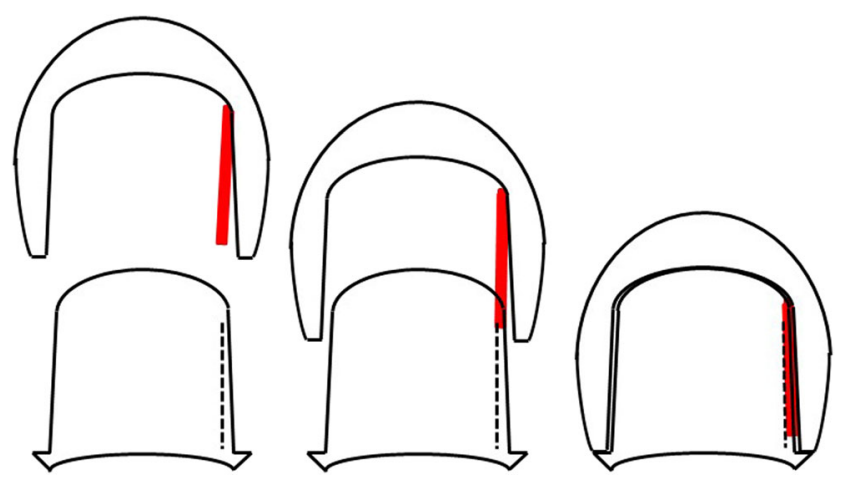

Fig. 1 Upgliding of the secondary crown with a friction pin (red) onto the primary crown into the study. Patients undergoing radiotherapy due to head and neck cancer were excluded because of their high risk of tooth decay [22].

The study protocol was approved by the Faculty of Medicine ethics committee at the Martin-Luther-University Halle-Wittenberg and conducted in accordance with the Declaration of Helsinki on Ethical Principles for Medical Research. The requirement for informed consent was waived, as this was a retrospective study.

\section{Pretreatment}

In accordance with the clinical guidelines of the Department of Prosthodontics at the Martin-Luther-University, all patients received a detailed clinical examination and screening. Conventional treatment, including preliminary periodontal treatment, build-up fillings, and functional preconditioning, was conducted where necessary.

\section{DCP denture fabrication}

The fabrication of DCP dentures was performed according to a standard protocol in the same dental laboratory (Rübeling+ Klar Dental-Labor, Berlin, Germany). Briefly, supporting teeth were prepared and a conventional tooth impression was performed using polyether (Impregum, Permadyne, 3M ESPE, Neuss, Germany). Wax template jaws were created, followed by a try in with a wax denture. Primary crowns were fabricated out of a cobalt-chrome-molybdenum alloy with a $2^{\circ}$ groove angle. The fit of the crown was controlled before the fixation impression. Next, the framework of the denture was fabricated, and friction pins were inserted. Passive fit was gained by the spark erosion procedure, where an insertion groove $\left(0^{\circ}\right)$ was placed in one approximal surface of the primary crown (Fig. 2). The corresponding friction pin $(\varnothing=0.7-$ $0.9 \mathrm{~mm}$ ) was fixed within the secondary crown by laser welding (Fig. 3) [17-21]. A final try in was performed to

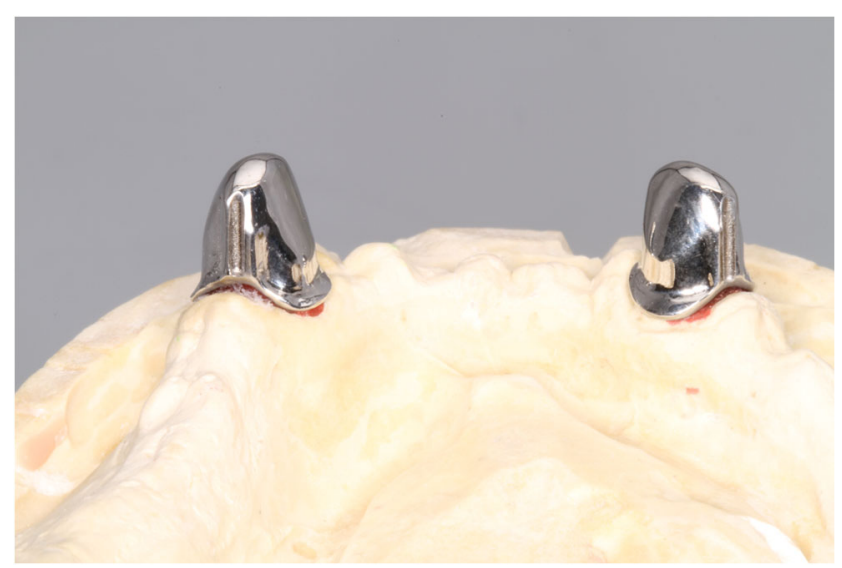

Fig. 2 Primary crowns with insertion groove $\left(0^{\circ}\right)$ on one approximal surface 


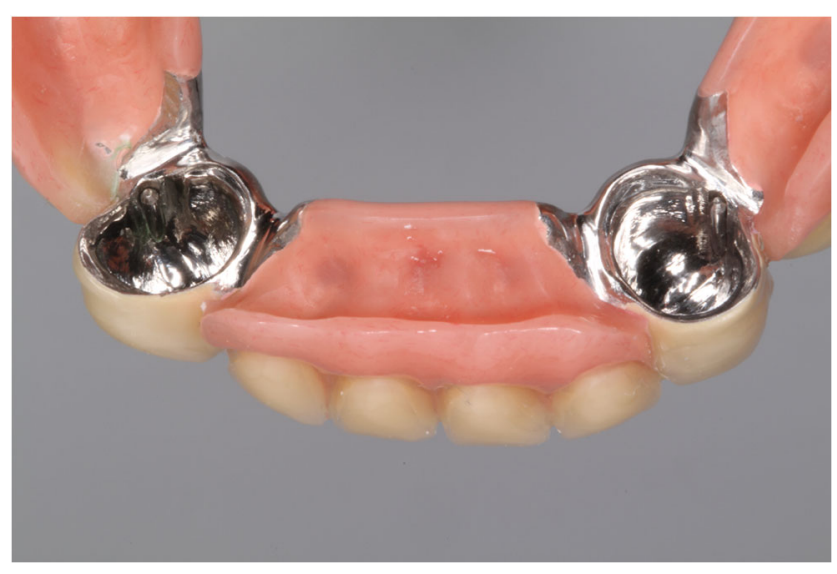

Fig. 3 Secondary crowns with corresponding friction pins, fixed by laser welding

assess jaw relation, occlusion, framework design, and esthetics. Significant emphasis was placed on a periodontalfriendly design and sufficient denture support. Primary crowns were fixed with zinc oxide phosphate cement (Hoffmann's CEMENT normal setting; Hoffmann Dental Manufaktur, Berlin, Germany) and the correct handling and maintenance of the dentures was explained to all patients.

\section{Patient follow-up}

All patients completed 6-month follow-up consultations. Subsequent dental checkups were arranged depending on patients' individual needs. Whenever possible, biannual recalls were performed and re-alignment was recommended where necessary. Existing dentures were modified in cases of tooth loss due to extraction or where decapitation of abutment teeth was required. Dental treatment or technical repairs were offered in cases of biological or technical failures.

\section{Statistical analysis}

Patients were divided according to the number of abutment teeth per denture: $>3$ teeth, not severely reduced dentitions (NSRD) and $\leq 3$ teeth, severely reduced dentitions (SRD). The distribution of abutment teeth was further categorized in accordance with Steffel's classification in the SRD group [12] (Fig. 4).

The DCP denture treatment start date was defined as the day of final denture insertion. DCP denture failure was defined as the day on which there was a loss of function due to final abutment tooth loss or technical failures necessitating remake of the denture. Abutment tooth failure was defined as the day on which there was a loss of function due to extraction (EX) or decapitation (DX). The last follow-up date was July 15, 2016, when the study was terminated. Anonymized data for survival analysis were acquired from the patient records.
Cumulative survival after 60 months was calculated using the Kaplan-Meier estimator with confidence intervals set to 95\% [23]. The influence of age, sex, jaw, number and localization of abutment teeth, and vitality, on the long-term survival of the dentures and abutment teeth was assessed over 96.5 months using the logrank test and Cox proportional hazards regression. Significance was set at $\alpha=0.05$. Analyses were performed using Microsoft Office Excel 2010 (Microsoft Corp., Redmond, USA) and IBM SPSS 25 (IBM Incorp., Armonk, USA).

\section{Results}

\section{Patient characteristics}

A total of 182 dentures, involving 520 abutment teeth, were provided for 158 patients $(55.1 \%$ male). The mean age on insertion was $62 \pm 12$ years (range, 24-87 years). The median observation time was 40 months (range, 1.4-96.5 months). Sixty-eight percent of all patients adhered to recommendations for biannual recalls.

\section{Denture and abutment tooth characteristics}

Denture and abutment tooth characteristics are provided in Table 1. A total of 61 abutment teeth were root filled, and 35 teeth underwent additional treatment with post and core. The abutment teeth consisted of 220 canines ( 85 maxilla/135 mandible), 159 premolars (62 maxilla/97 mandible), 79 incisors (68 maxilla/11 mandible), and 62 molars (47 maxilla/15 mandible). In the SRD group, 290 and 24 teeth were vital and nonvital, respectively. Eleven non-vital teeth were treated with a conventional root filling and post and core.

\section{Denture survival analysis}

The cumulative survival rate of the NSRD dentures was $100 \%$ after 60 months, and one denture was lost during the whole observation period. The cumulative survival rate of the SRD dentures was significantly lower than the NRSD dentures (80.3\%, CI $71.5-89.1 \%$, logrank $p=0.04)$ after 60 months; 13 dentures were modified into complete dentures $(\mathrm{CD})$, and five dentures had to be remade due to technical defects or loss (TD).

Denture loss in the SRD group was categorized using Steffel's classification: class A $=13(2 \mathrm{TD}, 11 \mathrm{CD})$; class $\mathrm{B}=6$ (3 TD, $3 \mathrm{CD})$; class $\mathrm{C} / \mathrm{D}=3(1 \mathrm{TD}, 2 \mathrm{CD})$; and class $\mathrm{E}=1(\mathrm{CD})$. Denture survival within the first 60 months is shown in Fig. 5. Class A showed a significantly lower survival rate than all other classes $(51.5 \%$; KI 30.9-72.1\%; $p<0.05)$. There was no difference in survival rate between class B (85.6\%, CI 69.8-100\%) and C/D (82.5\%, CI 63.5-100\%). 
Fig. 4 The Steffel classification of dentitions and residual support ( $\mathrm{A}=$ one remaining tooth, punctual support; $\mathrm{B}=$ two remaining teeth, linear sagittal support; $\mathrm{C} / \mathrm{D}=$ two remaining teeth, linear transversal/diagonal support; $\mathrm{E}=$ three remaining teeth, triangular support; class F (quadrangular support) is not shown)
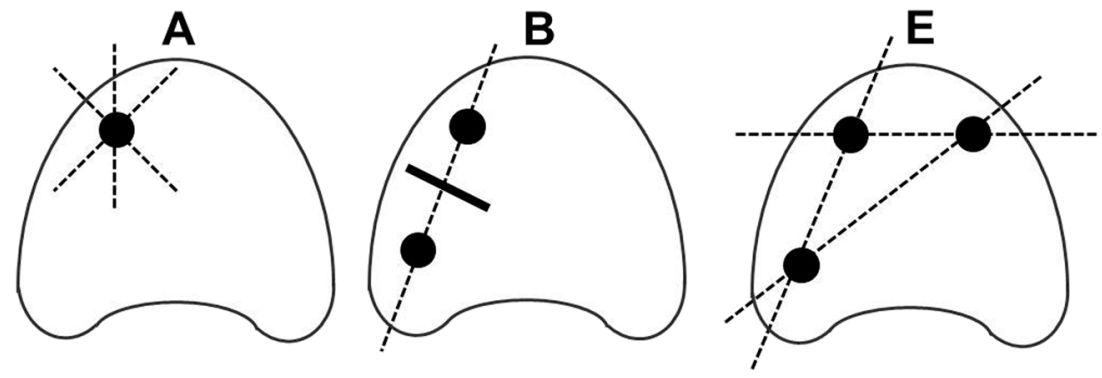

C / D

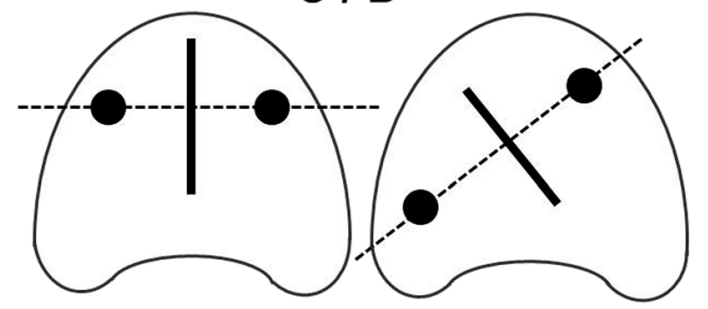

A- punctual support

B - linear sagittal support

C I D - linear transversal I diagonal support

E - triangular support
Effects of age, gender, and location on the survival rate were not found to be significant with $p$ values ranging between 0.144 and 0.792 .

For the multivariate Cox model, Steffel class A was chosen as the reference for the analysis of abutment tooth distribution. The estimated HRs and 95\% confidence intervals are presented in Table 2. Only Steffel class A was found to have a significant influence on denture survival.

\section{Abutment teeth survival analysis}

The cumulative survival of all abutment teeth was $83.74 \%$ (CI $80.8-94.4 \%$ ) after 60 months. There was a loss of 45 and 18 teeth in the $\operatorname{SRD}(n=314)$ and $\operatorname{NSRD}(n=459)$ group, respectively. The characteristics of abutment teeth loss are shown in Table 3.
Cumulative survival of abutment teeth in the NSRD group (87.6\%, CI 80.8-94.4\%) was higher than in the SRD group (80.8\%, CI 74.4-87.2\%; logrank $p=0.052$; Fig. 6). In the SRD group, abutment tooth loss was observed in the different groups as follows: class $\mathrm{A}=13$ (2 DX, $11 \mathrm{EX})$; class $\mathrm{B}=14$ (2 $\mathrm{DX}, 12 \mathrm{EX})$; class $\mathrm{C} / \mathrm{D}=6(\mathrm{EX})$; and class $\mathrm{E}=12(1 \mathrm{DX}, 11$ EX). The influence of abutment tooth distribution on tooth survival was significant $(p<0.001$; Fig. 7). Abutment teeth in the class $\mathrm{E}$ group showed the highest survival rates (91.6\%; (CI 84.8-98.4\%), followed by class C/D (80.8\%, CI 66.2-95.4\%), and class B (78.2\%, CI 65.4-91\%). Abutment teeth in the class A group showed the lowest survival rates $(51.5 \%$, CI $30.9-72.1 \%)$. There were no differences in abutment teeth survival between classes B-E $(p=0.333)$.

Tooth type had no significant influence on survival in the SRD group $(p=0.957)$. Survival rates were calculated as follows: molars, $87.2 \%$ (CI 73.2-100\%); premolars, $84.4 \%$ (CI
Table 1 Denture and abutment teeth characteristics

\begin{tabular}{|c|c|c|c|c|c|c|}
\hline & \multicolumn{5}{|l|}{ SRD } & \multirow[t]{2}{*}{ NSRD } \\
\hline & Total & Steffel class A & Steffel class B & Steffel class $\mathrm{C} / \mathrm{D}$ & Steffel class E & \\
\hline Dentures & \multirow[t]{2}{*}{144} & \multirow[t]{2}{*}{30} & \multirow[t]{2}{*}{36} & \multirow[t]{2}{*}{34} & \multirow[t]{2}{*}{44} & \multirow[t]{2}{*}{38} \\
\hline$n=182$ & & & & & & \\
\hline Maxilla & \multirow[t]{2}{*}{73} & \multirow[t]{2}{*}{18} & \multirow[t]{2}{*}{21} & \multirow[t]{2}{*}{16} & \multirow[t]{2}{*}{18} & \multirow[t]{2}{*}{17} \\
\hline$n=90$ & & & & & & \\
\hline Mandible & \multirow[t]{2}{*}{71} & \multirow[t]{2}{*}{12} & \multirow[t]{2}{*}{15} & \multirow[t]{2}{*}{18} & \multirow[t]{2}{*}{26} & \multirow[t]{2}{*}{21} \\
\hline$n=92$ & & & & & & \\
\hline Abutment teeth & \multirow[t]{2}{*}{314} & \multirow[t]{2}{*}{30} & \multirow[t]{2}{*}{84} & \multirow[t]{2}{*}{68} & \multirow[t]{2}{*}{132} & \multirow[t]{2}{*}{206} \\
\hline$n=520$ & & & & & & \\
\hline Maxilla & \multirow[t]{2}{*}{154} & \multirow[t]{2}{*}{18} & \multirow[t]{2}{*}{50} & \multirow[t]{2}{*}{32} & \multirow[t]{2}{*}{54} & \multirow[t]{2}{*}{108} \\
\hline$n=262$ & & & & & & \\
\hline Mandible & \multirow[t]{2}{*}{160} & \multirow[t]{2}{*}{12} & \multirow[t]{2}{*}{34} & \multirow[t]{2}{*}{36} & \multirow[t]{2}{*}{78} & \multirow[t]{2}{*}{98} \\
\hline$n=258$ & & & & & & \\
\hline
\end{tabular}

$N S R D$, not severely reduced dentition; $S R D$, severely reduced dentition 
Fig. 5 Kaplan-Meier survival curves of dentures in the NSRD group and Steffel classes (NSRD, not severely reduced dentition)

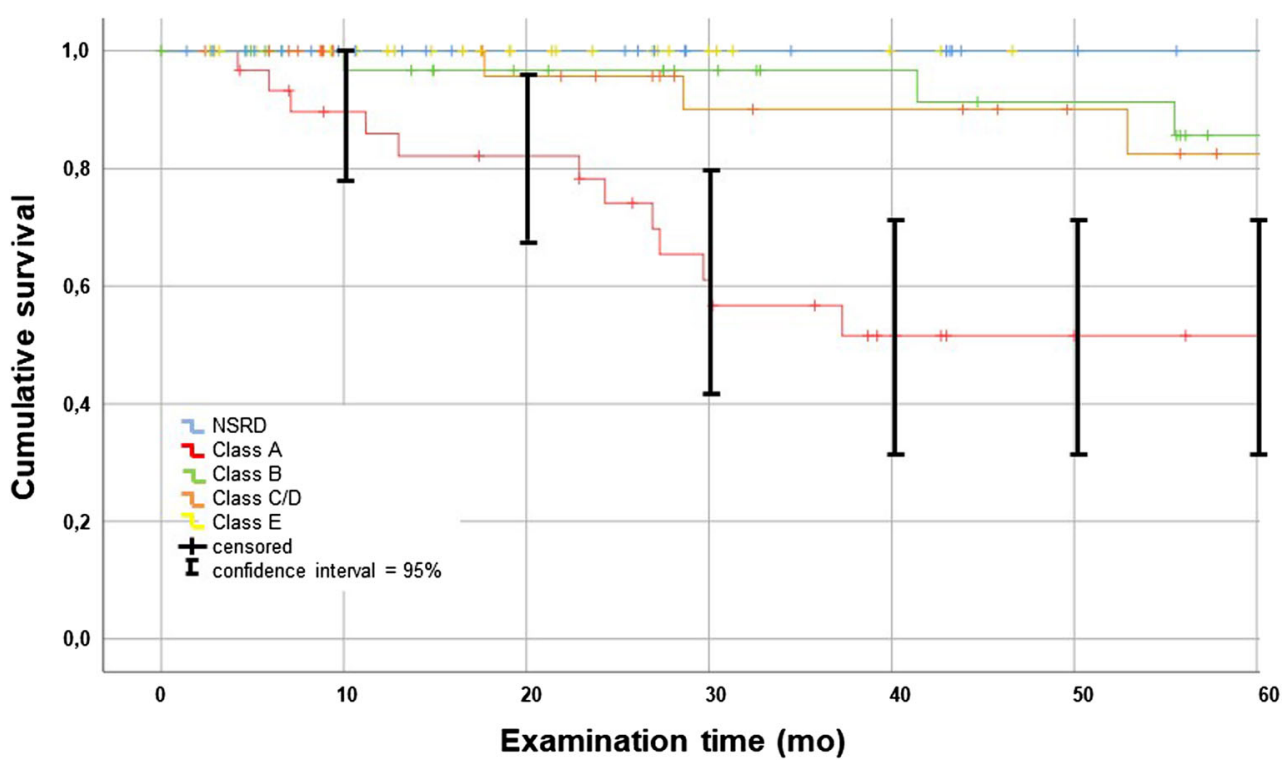

73.8-95\%); incisors, $84.3 \%$ (CI 67.1-100\%); and canines, $76.4 \%$ (CI 66.6-86.2\%). Furthermore, tooth type had no significant influence on survival $(p=0.944)$ in the NSRD group. Survival rates were calculated as follows: molars, $95.5 \%$ (CI 86.7-100\%); premolars, $94.1 \%$ (CI 85.7-100\%), canines, $80.1 \%$ (CI 63.7-96.5\%); and incisors, $83.3 \%$ (CI 69.1$97.5 \%$ ). Survival of maxillary abutment teeth did not differ significantly compared with mandibular abutment teeth $(p>0.05)$.

Vital abutment teeth showed higher survival rates $(84.6 \%$, CI 78.8-90.4\%) than teeth with root canal fillings and posts (58.3\%, CI $21.1-95.5 \%)$ or root canal fillings alone (35.6\% (CI $0-73 \%)$ in the SRD group $(p<0.001)$. In the NSRD group, vital abutment teeth had slightly higher survival rates (90.9\%, CI 84.5-97.3\%) than teeth with root canal fillings and post and core $(75.1 \%$, CI 54.9-95.3\%) or root canal fillings alone $(77.4 \%$, CI $47-100 \% ; p=0.314)$. The survival rate of teeth with root canal fillings alone, and teeth with an additional post and core, did not differ significantly $(p>0.05)$.

For the multivariate Cox model, incisors were chosen as the reference for tooth type, and Steffel class A was chosen as the reference for analysis of abutment tooth distribution. The estimated HRs and 95\% confidence intervals are presented in Table 4. Patient age, sex, and both abutment tooth vitality and distribution were found to have a significant influence on abutment tooth survival.

\section{Technical complications}

Over the course of the observation period, 33 cases of primary crown loosening (6.3\% of all primary crowns) were documented. All crowns were re-cemented. In 22 of 182 DCP dentures $(12.1 \%)$, friction pins were activated after insertion in order to increase denture retention. In 7 cases (3.8\%), friction pins were deactivated. In 7 secondary crowns $(1.3 \%)$, loss of the friction pin was documented due to fracture of the laserwelded connection between the friction pin and the metal framework of the secondary crown.

\section{Discussion}

The aim of this study was to evaluate the long-term survival of DCP dentures. To the best of our knowledge, this is the first
Table 2 DCP dentures: hazard ratios of the different variables calculated with multivariate analysis

\begin{tabular}{lllll}
\hline Variable & & Hazard ratio & $95 \%$ CI & $p$ value \\
\hline Age & & 1.029 & $0.989-1.071$ & 0.154 \\
Sex & Male vs. female & 0.801 & $0.336-1.912$ & 0.617 \\
Jaw & Maxilla vs. mandible & 0.726 & $0.302-1.746$ & 0.474 \\
Tooth distribution & Class A vs. B & 0.185 & $0.061-0.559$ & 0.003 \\
& Class A vs. C/D & 0.137 & $0.036-0.517$ & 0.003 \\
& Class A vs. E & 0.022 & $0.003-0.187$ & $<0.001$ \\
& Class A vs. NSDR & 0.029 & $0.004-0.229$ & 0.001 \\
\hline
\end{tabular}


Table 3 Characteristics of abutment tooth failure

\begin{tabular}{|c|c|c|c|c|c|c|c|c|c|c|c|c|}
\hline \multirow[b]{2}{*}{ Tooth type } & \multirow[b]{2}{*}{ Therapy } & \multicolumn{2}{|c|}{ Fracture } & \multicolumn{2}{|c|}{ Periodontitis } & \multicolumn{2}{|c|}{ Endodontic problems } & \multicolumn{2}{|l|}{ Caries } & \multicolumn{2}{|c|}{ Unknown } & \multirow[t]{2}{*}{ Total } \\
\hline & & NSRD & SRD & NSRD & SRD & NSRD & SRD & NSRD & SRD & NSRD & SRD & \\
\hline \multirow[t]{2}{*}{ Incisors } & EX: & 3 & 3 & 0 & 0 & 0 & 2 & 1 & 0 & 1 & 0 & 10 \\
\hline & DX: & 1 & 0 & 0 & 0 & 0 & 0 & 0 & 0 & 0 & 0 & 1 \\
\hline \multirow[t]{2}{*}{ Canines } & EX: & 1 & 11 & 1 & 3 & 1 & 3 & 0 & 1 & 0 & 2 & 23 \\
\hline & DX: & 2 & 3 & 0 & 0 & 0 & 0 & 0 & 0 & 0 & 0 & 5 \\
\hline \multirow[t]{2}{*}{ Premolars } & EX: & 0 & 6 & 0 & 2 & 2 & 1 & 1 & 0 & 0 & 1 & 13 \\
\hline & DX: & 2 & 2 & 0 & 0 & 0 & 0 & 0 & 0 & 0 & 0 & 4 \\
\hline \multirow[t]{2}{*}{ Molars } & EX: & 1 & 2 & 0 & 2 & 0 & 0 & 1 & 1 & 0 & 0 & 7 \\
\hline & DX: & 0 & 0 & 0 & 0 & 0 & 0 & 0 & 0 & 0 & 0 & 0 \\
\hline
\end{tabular}

clinical study to analyze DCP denture survival over 60 months. The number of patients participating in this study is comparable with other clinical studies assessing the longterm survival of different attachments, indicating that our results can be compared with these investigations $[11,13$, 24-26].

The survival rate of DCP dentures was $84.3 \%$ after 60 months in this study. Previous studies have reported higher survival rates, such as $95.1 \%$ survival for dentures fixed on telescopic crowns [3]. Schwindling et al. investigated different types of double crowns and found general survival rates of $96.5 \%$, with $96.4 \%$ survival when using galvano crowns [24, $25]$. Ishida et al. have reported $100 \%$ survival; however, they did not specify the type of double crown used [27]. The higher survival rates may be due to a lower proportion of SRD cases included in previous studies. In addition, most studies did not perform residual dentition classification, and a lower proportion of cases defined as Steffel class A might have a positive influence on denture survival rates. Furthermore, the proportion of non-vital abutment teeth has a decisive influence on denture survival rate [28], and previous studies may have included a lower proportion of these teeth. An additional difference may be due to the current study classifying both a modification of DCP dentures to complete dentures (13 cases), and denture destruction or loss (6 cases), as failure. The survival rate of all DCP dentures in the first 60 months would have been otherwise increased to $88.9 \%$ (CI 82.9-94.9\%).

We found that DCP dentures in the NSRD group had a better survival rate than the SRD group, which is most likely due to the greater number and more favorable distribution of abutment teeth, resulting in better denture support. Survival rates in this study were comparable with data in the literature [13]. The survival rates of dentures in Steffel classes B-E were comparable with dentures in the NSRD group. Interestingly, class E dentures had higher survival rates than NSRD dentures. This may be due to more consistent and homogenous
Fig. 6 Kaplan-Meier survival curves of abutment teeth in the NSRD and SRD groups (NSRD, not severely reduced dentition; $\mathrm{SRD}$, severely reduced dentition)

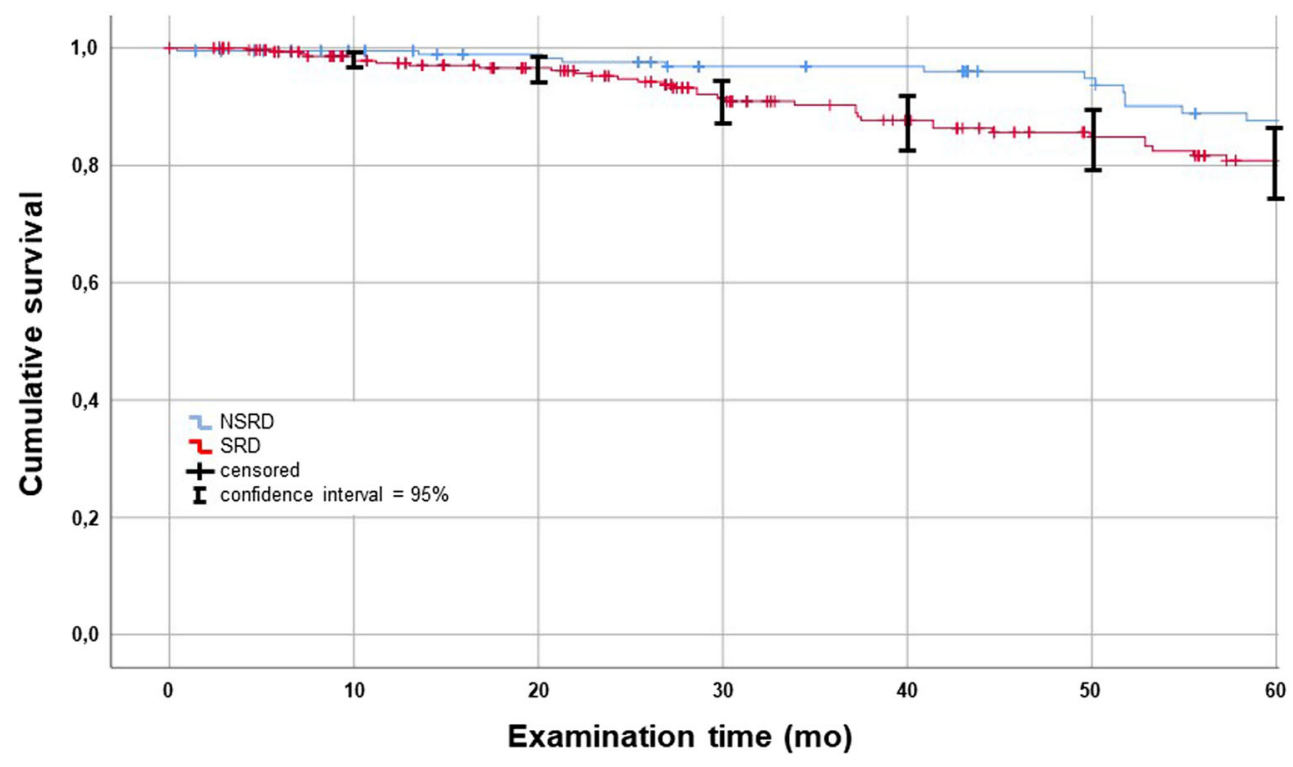


Fig. 7 Kaplan-Meier survival curves of abutment teeth in the NSRD group and Steffel classes (NSRD, not severely reduced dentition)

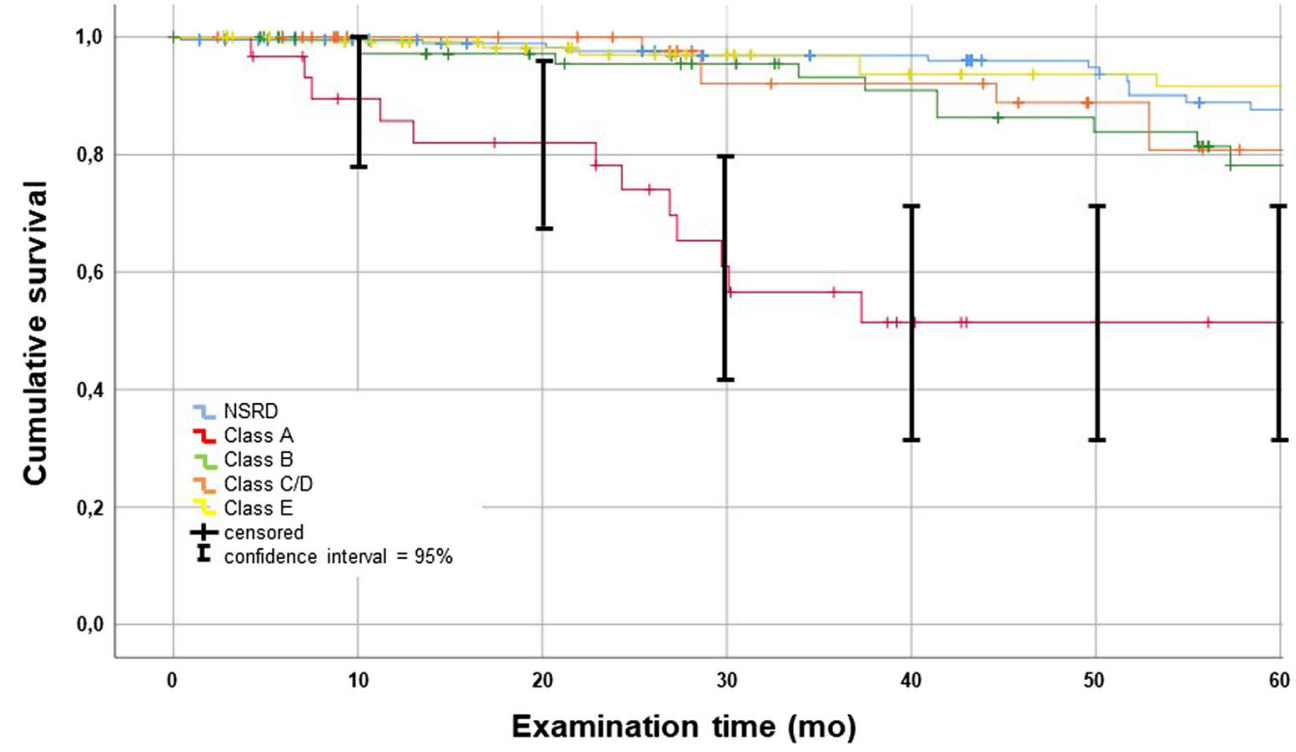

force distribution in everyday use in triangular supported dentures that compensates for an overload on single teeth. Moreover, we have shown that the positive prognosis of dentures supported by two abutment teeth is increased when compared with dentures that are supported by a single tooth. These data are in line with a previous study that reports survival rates of $70.9 \%$ for one abutment tooth, $90.4 \%$ for two abutment teeth, and $95 \%$ for three abutment teeth over 5 years in dentures fixed with telescopic crowns [3].

In order to assess the influence of specific parameters on DCP denture survival, a cox regression was performed. Denture survival was found to be solely dependent on the number and localization of the abutment teeth, with the worst results yielded for Steffel class A. Patient age, sex, and the arch treated did not have a significant influence on denture survival.

The survival rate of all abutment teeth was $83.4 \%$ after 60 months, which is similar to the survival rate of the abutment teeth treated using the Marburg double crown method
$[26,29]$. This denture is created from a cobalt-chromiummolybdenum alloy with an additional retention element within the secondary crown, which is an elastically fixed ball with a corresponding deepening in the primary crown (TC.SNAP, Sitec, Herdecke, Germany) [26, 29].

The survival rate of abutment teeth was lower in the NSRD group than the SRD group. Wenz and Lehmann found survival rates of $89 \%$ and $97 \%$ for SRD and NSRD groups, respectively, after 60 months [26]. However, they did not use Steffel's classification to characterize the SRD group. We found that abutment teeth of classes B-E had comparable survival rates to NSRD abutment teeth, with the highest and lowest survival rates belonging to abutment teeth of classes $\mathrm{E}$ and A, respectively. Szentpétery et al. used Steffel's classification and found comparable survival rates [11]. The authors suggested that the survival rates of class A abutment teeth may be due to regular and thorough aftercare. Due to the retrospective character of the current study, it was not possible to assess this; thus, our study might better reflect clinical reality.
Table 4 Abutment teeth: hazard ratios of the different variables calculated with multivariate analysis

\begin{tabular}{lllll}
\hline Variable & & Hazard ratio & $95 \%$ CI & $p$ value \\
\hline Age & & 1.034 & $1.011-1.058$ & 0.004 \\
Sex & Male vs. female & 2.075 & $1.197-3.598$ & 0.009 \\
Jaw & Maxilla vs. mandible & 0.845 & $0.478-1.495$ & 0.563 \\
Tooth vitality & Vital vs. non-vital & 2.973 & $1.628-5.427$ & $<0.001$ \\
Tooth type & Incisors vs. canines & 0.711 & $0.310-1.631$ & 0.420 \\
& Incisors vs. premolars & 0.704 & $0.309-1.603$ & 0.403 \\
& Incisors vs. molars & 0.499 & $0.179-1.395$ & 0.185 \\
Tooth distribution & Class A vs. B & 0.124 & $0.049-0.313$ & $<0.001$ \\
& Class A vs. C/D & 0.098 & $0.035-0.274$ & $<0.001$ \\
& Class A vs. E & 0.062 & $0.024-0.156$ & $<0.001$ \\
& Class A vs. NSDR & 0.081 & $0.035-0.185$ & $<0.001$ \\
\hline
\end{tabular}


Therefore, we suggest that the higher survival rates of class E abutment teeth are more likely to be due to statistical distribution, as postulated by Szentpétery et al. [11]. However, the influence of the double crown type on survival rate seems to be comparably low, as indicated by similar results between studies using different double crown types and materials.

Tooth type did not have a significant influence on survival rate. In both groups, the highest survival rates were found in molars. This is in line with previous results [11]. Higher survival may be due to the increased proportion of canines $(n=$ $220)$ used as abutment teeth when compared with molars $(n=$ 62). The fracture rate was $7.7 \%$ and $9.3 \%$ in canines and molars, respectively; therefore, if more molars were integrated into the treatment, tooth loss may have been similar between groups. Furthermore, the requirement for better prosthesis support often requires the inclusion of molars as abutment teeth with anterior teeth. Canines are often chosen as the abutment teeth when all other teeth have been lost. Thus, they may have to withstand higher leverage from the denture.

In order to weight all parameters investigated concerning their influence on both DCP denture and abutment tooth survival, an additional Cox regression was performed. Besides tooth distribution, age, sex, and abutment tooth vitality also had a significant influence on abutment tooth survival. Several studies have found that vital abutment teeth can provide up to double the survival rate when compared with non-vital teeth $[3,11,30,31]$. However, concerning the parameters sex and jaw, Szentpétery et al. also found that telescopic crowns located in female mandibles provide higher survival rates than in males [11]. This is in contrast to the results of our study, and the discrepancy may have been due to differences in the study population and the proportion of SRDs included. Survival rate was found to decrease with increasing patient age. This may not have been surprising, given the reduction of manual dexterity and associated difficulties with oral hygiene observed in older patients. Elders have also been shown to have more difficulties with denture removal and insertion, thus increasing the risk of biological or technical complications.

Regarding the results of our study, several limitations have to be taken into account. Firstly, all the dentures evaluated were created using the same procedures in the same dental laboratory; however, the dental treatment was performed by different dentists with different levels of clinical experience. Moreover, follow-up treatment and denture adjustments were performed by different dentists, and follow-up intervals differed due to patient non-compliance. In contrast to other studies, we cannot guarantee equal levels of treatment throughout the study. Secondly, data were analyzed retrospectively based on the patients' records. Wöstmann et al. found that a regular recall system increases abutment tooth survival rate [3]. While a biannual recall was intended in our study, $32 \%$ of patients did not adhere to this recommendation. Thus, if regular control and aftercare would have been performed, abutment tooth survival rate might have been improved. Thirdly, 21 patients received DCP dentures in both the maxilla and the mandible. This aspect might have been a confounder which was not considered in our analysis.

\section{Conclusion}

DCP dentures provide an acceptable survival rate after 60 months in both SRD and NSRD groups. As an advantage, activation of the friction pin can be performed easily without effort. In the severely reduced dentition, longevity mainly depends on the number and distribution of the abutment teeth. Generally, non-vital abutment teeth provide a lower likelihood of survival than vital teeth. Nevertheless, the lower success rate of DCP dentures in patients with SRDs needs to be placed in the context of each individual patient's oral condition and treatment need. This should also include an evaluation of the potential functional benefits gained from the prosthesis, even though this may likely be over a relatively shorter period of clinical use.

Funding Information Open Access funding provided by Projekt DEAL.

\section{Compliance with ethical standards}

Conflict of interest The authors declare that they have no conflict of interest.

Ethical approval The study protocols were approved by the medical faculty's ethics committee at the Martin-Luther-University HalleWittenberg and conducted in accordance with the Declaration of Helsinki on Ethical Principles for Medical Research.

Informed consent For this type of study, formal consent was not required.

Open Access This article is licensed under a Creative Commons Attribution 4.0 International License, which permits use, sharing, adaptation, distribution and reproduction in any medium or format, as long as you give appropriate credit to the original author(s) and the source, provide a link to the Creative Commons licence, and indicate if changes were made. The images or other third party material in this article are included in the article's Creative Commons licence, unless indicated otherwise in a credit line to the material. If material is not included in the article's Creative Commons licence and your intended use is not permitted by statutory regulation or exceeds the permitted use, you will need to obtain permission directly from the copyright holder. To view a copy of this licence, visit http://creativecommons.org/licenses/by/4.0/.

\section{References}

1. Arnold C, Hey J, Setz JM, Boeckler AF, Schweyen R (2018) Retention force of removable partial dentures with different double crowns. Clin Oral Invest 22:1841-1649 
2. Rinke S, Ziebolz D, Ratka-Krüger P, Frisch E (2015) Clinical outcome of double crown-retained mandibular removable dentures supported by a combination of residual teeth and strategic implants. J Prosthodont 24:358-365

3. Wöstmann B, Balkenhol M, Weber A, Ferger P, Rehmann P (2007) Long-term analysis of telescopic crown retained removable partial dentures: survival and need for maintenance. J Dent 35:939-945

4. Majcher A, Lesniewska-Kochanek A, Mierzwinska-Nastalska E (2017) A method and a device for the evaluation of the retention of telescopic dental crowns. J Mech Behav Biomed Mater 69:362 367

5. Cho JH, Cho SA (2016) The use of telescopic crowns in removable partial denture treatment for patients with severe periodontal disease: two patient case history reports. Int J Prosthodont 29:175-178

6. Bhagat TV, Walke AN (2015) Telescopic partial denturesconcealed technology. J Int Oral Health 7:143-147

7. Gupta SH, Viswambaran M, Vijayakumar R (2015) Telescopic retainers for removable partial dentures. Med J Armed Forces India 71:S578-S580

8. Zoidis P, Panagiota S, Polyzois G (2015) A fixed telescopic prosthesis designed to retrieve and convert to fixed-removable combination case: a clinical report. Acta Stomatol Croat 49:145-150

9. Wadhwa B, Jain V, Pruthi G (2014) Strategic use of telescopic retainers and semi-rigid precision attachments in a geriatric patient: a case report. J Indian Prosthodont Soc 14:S232-S237

10. Szentpétery V, Lautenschlager C, Setz JM (2010) Longevity of frictional telescopic crowns in the severely reduced dentition: 3year results of a longitudinal prospective clinical study. Quintessence Int 41:749-758

11. Szentpétery V, Lautenschlager C, Setz JM (2012) Frictional telescopic crowns in severely reduced dentitions: a 5 -year clinical outcome study. Int J Prosthodont 25:217-220

12. Steffel VL (1962) Planning removable partial dentures. J Prosthet Dent 12:524-535

13. Verma R, Joda T, Brägger U, Wittneben JG (2013) A systematic review of the clinical performance of tooth-retained and implantretained double crown prostheses with a follow-up of $\geq 3$ years. $\mathrm{J}$ Prosthodont 22:2-12

14. Bayer S, Kraus D, Keilig L, Gölz L, Stark H, Enkling N (2012) Changes in retention forces with electroplated copings on conical crowns: a comparison of gold and zirconia primary crowns. Int $\mathrm{J}$ Oral Maxillo Implants 27:577-585

15. Bayer S, Stark H, Gölz L, Keilig L, Kraus D, Hansen A, Enkling N (2012) Clinical retention force development of double crowns. Clin Oral Invest 16:407-411

16. Bayer S, Zuziak W, Kraus D, Keilig L, Stark H, Enkling N (2011) Conical crowns with electroplated gold copings: retention force changes caused by wear and combined off-axial load. Clin Oral Impl Res 22:323-329

17. Jo LJ (2011) Spark erosion process: an overview. J Dental Implants $1: 2-6$

18. Eisenmann E, Mokabberi A, Walter MH, Freesmeyer WB (2004) Improving the fit of implant-supported superstructures using the spark erosion technique. Int J Oral Maxillofac Implants 19(6): 810-818

19. Rübeling G (1999) New techniques in spark erosion: the solution to an accurately fitting screw-retained implant restoration. Quintessence Int 30(1):37-48

20. Weber H, Frank G (1993) Spark erosion procedure: a method for extensive combined fixed and removable prosthodontic care. J Prosthet Dent 69:222-227

21. Rübeling G, Kreylos HA (1984) Spark erosion in dental technology: possibilities and limitations. Quintessence Dent Tech 8:649657

22. Schweyen R, Hey J, Fränzel W, Vordermark D, Hildebrandt G, Kuhnt T (2012) Radiation-related caries: etiology and possible preventive strategies. What should the radiotherapist know? Strahlenther Onkol 188:21-28

23. Pocock SJ, McMurray JJV, Collier TJ (2015) Making sense of statistics in clinical trial reports part 1 of a 4-part series on statistics for clinical trials. J Am Coll Cardiol 66:2536-2549

24. Schwindling FS, Dittmann B, Rammelsberg P (2014) Doublecrown-retained removable dental prostheses: a retrospective study of survival and complications. J Prosthet Dent 112: 488-493

25. Schwindling FS, Lehmann F, Terebesi S, Corcodel N, Zenthöfer A, Rammelsberg P, Stober T (2017) Electroplated telescopic retainers with zirconia primary crowns: 3 -year results from a randomized clinical trial. Clin Oral Invest 21:2653-2660

26. Wenz HJ, Hertrampf K, Lehmann KM (2001) Clinical longevity of removable partial dentures retained by telescopic crowns: outcome of the double crown with clearance fit. Int J Prosthodont 14:207213

27. Ishida K, Nogawa T, Takayama Y, Saito M, Yokoyama A (2017) Prognosis of double crown-retained removable dental prostheses compared with clasp-retained removable dental prostheses: a retrospective study. J Prosthodont Res 61:268-275

28. Wegner PK, Freitag S, Kern M (2006) Survival rate of endodontically treated teeth with posts after prosthetic restoration. J Endod 32:928-931

29. Wenz H, Lehmann KM (1998) A telescopic crown concept for the restoration of the partially edentulous arch: the Marburg double crown system. Int J Prosthodont 11:541-550

30. Stober T, Bermejo JL, Beck-Mußoter J, Seche AC, Lehmann F, Koob J, Rammelsberg P (2012) Clinical performance of conical and electroplated telescopic double crown-retained partial dentures: a randomized clinical study. Int J Prosthodont 25:209-216

31. Dittmann B, Rammelsberg P (2008) Survival of abutment teeth used for telescopic abutment retainers in removable partial dentures. Int J Prosthodont 21:319-321

Publisher's note Springer Nature remains neutral with regard to jurisdictional claims in published maps and institutional affiliations. 